\title{
Formation of the Liquid Crystals of Poly(L-lysinehydrobromide) in Aqueous Solution
}

\author{
Yoshiyuki Kondo, Yoshiro UkaI, and Eisaku IIzUKa \\ Institute of High Polymer Research, Faculty of Textile Science \\ and Technology, Shinshu University, Ueda 386, Japan.
}

(Received January 31, 1978)

\begin{abstract}
KEY WORDS Poly(L-lysinehydrobromide) / Lyotropic Liquid Crystal /
Coil-to-Helix Transition / Molecular Cluster /
\end{abstract}

In a previous paper, ${ }^{1}$ we have reported that at a certain polymer concentration sodium salt of poly(glutamic acid) (PGA-Na) undergoes a coilto-helix transition and eventually becomes liquid crystalline. In expectation of obtaining liquid crystals of polypeptides in the $\beta$ conformation, the properties of poly(L-lysinehydrobromide) $(\mathrm{PLL} \cdot \mathrm{HBr})$ in a concentrated aqueous solution have been investigated. When the solution of this polypeptide is heated up to $50^{\circ} \mathrm{C}$ at an alkaline $\mathrm{pH}$ of 11 , the polypeptide molecules turn into the $\beta$ conformation and precipitate. Thus, attempts to obtain any liquid crystalline structure in the $\beta$ conformation have been unsuccessful; however, at acidic pHs liquid crystals which are quite similar to those found in PGA-Na have been obtained. We wish to report the formation of such liquid

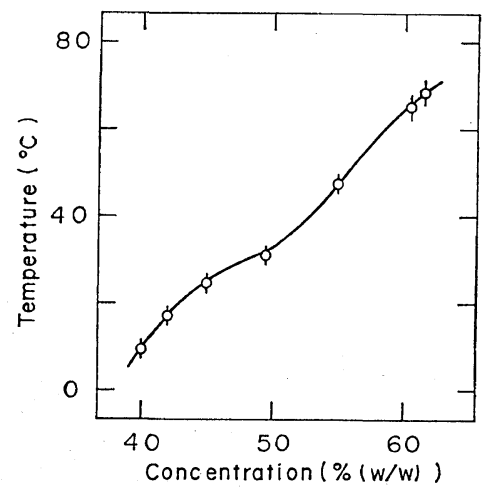

Figure 1. Polymer concentration dependence of the isotropic-to-birefringent transition temperature of $\mathrm{PLL} \cdot \mathrm{HBr}$. crystals in this note.

The solution of PLL $\cdot \mathrm{HBr}$ becomes slightly turbid at a certain temperature that depends on the polymer concentration and eventually undergoes an isotropic-to-birefringent transition (Figure 1). The concentration of this polymer solution which undergoes such a transition at room temperature $\left(22^{\circ} \mathrm{C}\right)$ is $45 \mathrm{wt} \%$. The amide-II band of the polypeptide molecules dissolved in $\mathrm{D}_{2} \mathrm{O}$ to arrange the polymer concentration above this critical concentration appears both at $1540 \mathrm{~cm}^{-1}$ and at $1445 \mathrm{~cm}^{-1}$, unlike those dissolved to make the polymer concentration below the critical concentration, which have only one amide-II band, at $1445 \mathrm{~cm}^{-1}$ (Figure 2). This suggests that in the

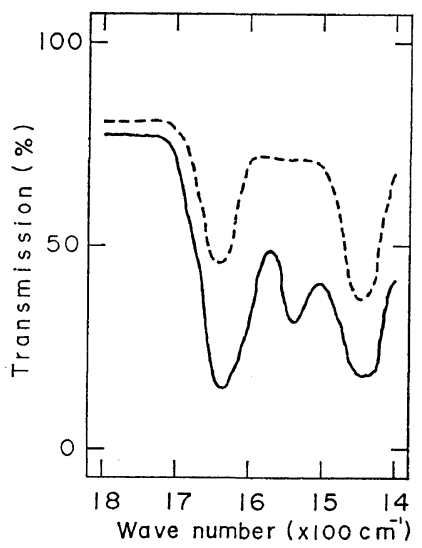

Figure 2. Infrared absorption spectra of PLL $\cdot \mathrm{HBr}$. Measured in $\mathrm{D}_{2} \mathrm{O}$. Solid line, the birefringent solution $(47 \mathrm{wt} \%$ ); broken line, the isotropic solution (40 wt $\%)$. 
birefringent solution the peptide groups of this polymer molecules are not completely deuterated, as they would be in the isotropic solution, and that the polymer molecules are in some ordered conformation. The amide-I band of the isotropic solution is found at $1645 \mathrm{~cm}^{-1}$ and that of the birefringent solution at $1640 \mathrm{~cm}^{-1}$, making a slight shift to a shorter wave number. No absorption maximum centered at $1620-1630 \mathrm{~cm}^{-1}$, typical of the $\beta$ form is observed. These results suggest that the polymer molecules are most likely in the $\alpha$-helical conformation in the birefringent solution. $^{2}$ In a dilute solution of PLL. $\mathrm{HBr}$, the amino groups of the polypeptide molecules are completely ionized at acidic pHs and the polymer chains are well extended. ${ }^{3}$ The increase of polymer concentration would cause the decrease of thermodynamic activity of the solvent water molecules by modifying solvent-polymer interactions ${ }^{4}$ in a similar manner as in PGA-Na ${ }^{1}$ and poly $(\gamma$-benzyl L-glutamate) in dichloroacetic $\operatorname{acid}^{5}$ to invite a coil-to-helix transition.

The birefringent solution can very easily be oriented by stresses that shear the solution during the insertion of a spacer into the optical cell. Therefore, the pattern under crossed polaroids differs from solution to solution (Figure 3, a-c); lines are observed always parallel to the shear direction. The pattern is also dependent on the polymer concentration, suggesting that some change of solution structure has occurred; at the polymer concentration of $75 \mathrm{wt} \%$ or more, the pattern suggests the formation of some crystalline (or semicrystalline) structure in solution (Figure 3, e and f). However, experiments have been unable to determine a critical polymer concentration at which such a transition occurs, since the change of the pattern is successive. The small-angle laser light scattering pattern of the birefringent solution taken at crossed polaroids ( $H_{\nabla}$ pattern) is always of the $\pm 45^{\circ}$ type when the polymer concentration is $70 \mathrm{wt} \%$ or less, suggesting that the birefringent solution is a collection of rod-like molecular assemblies (molecular clusters) of the polymer molecules in which the maximum polarizability directions are parallel or perpendicular to the long rod-axes ${ }^{6,7}$ (Figure 4). The presence of such molecular clusters was first found with poly( $\gamma$-benzyl L-glutamate). ${ }^{8,9}$ When the solution is sheared up and down, the upper (and the lower) angles of intersection of the cross pattern $\left( \pm 45^{\circ}\right.$ type pattern) become larger than $90^{\circ}$ and the $V_{\nabla}$ pattern is long transversely rather than circular (Figure 4, a). These results suggest that the mole-

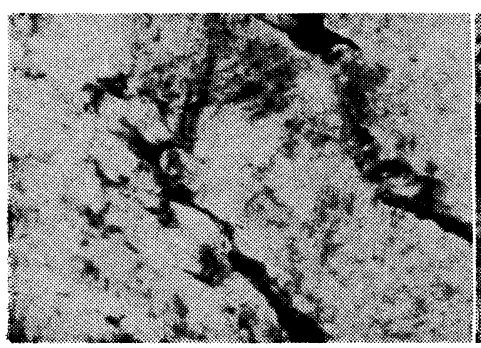

a

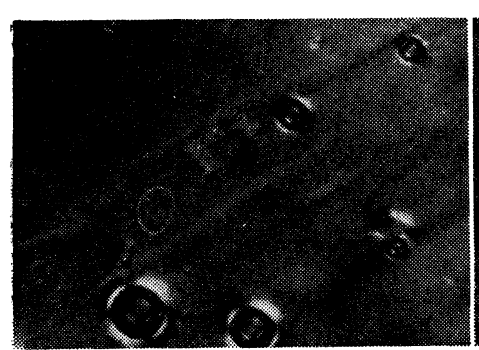

d

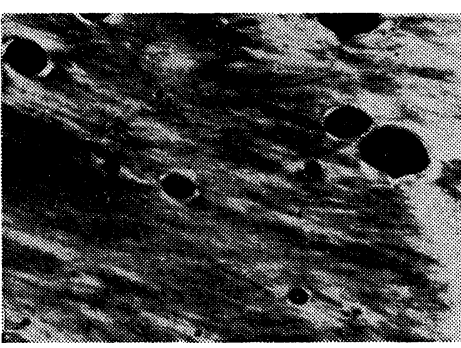

b

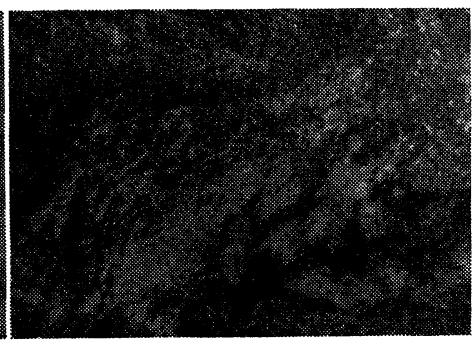

e

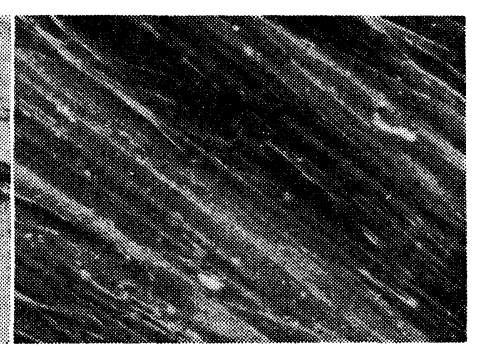

c

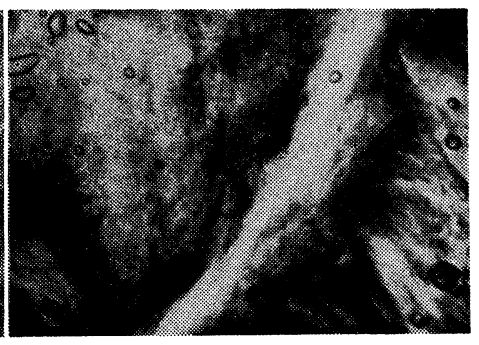

f

Figure 3. Polarizing micrographs of the concentrated solutions of PLL $\cdot \mathrm{HBr}$. Polymer concentration in wt $\%: a-c, 48 ; d, 60 ; e, 75 ; f, 85$. Lines appear in the shear direction in $c$. 

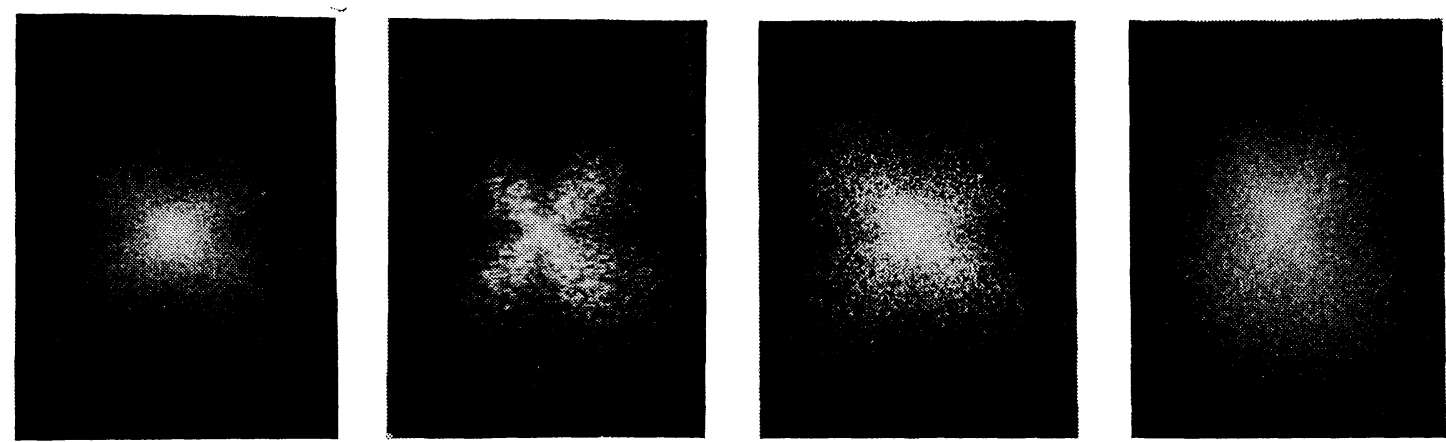

$-3^{\circ}$

$-2^{\circ}$

$-1^{\circ}$

$-$

$\frac{0}{5}$

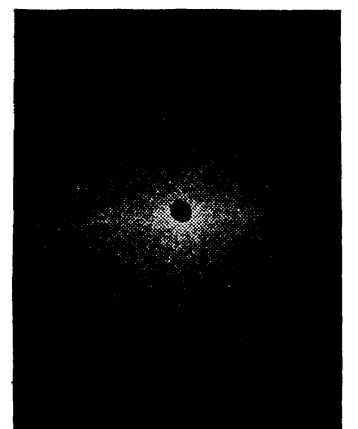

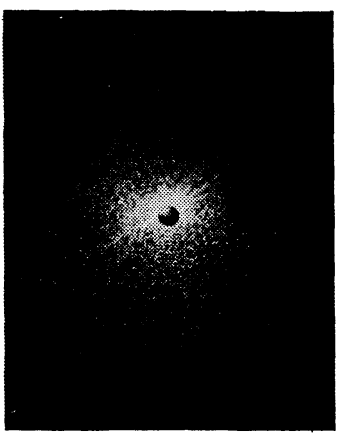

b

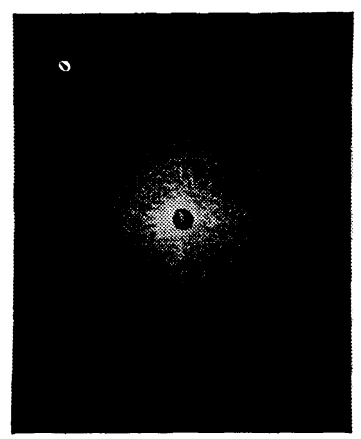

C

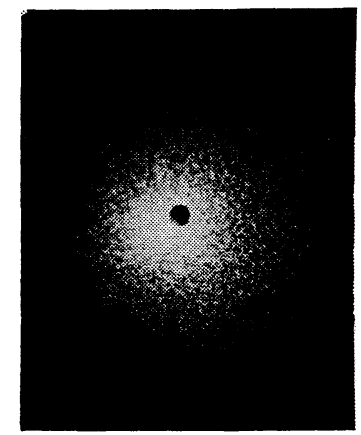

$-3^{\circ}$

$-2^{\circ}$

$-1^{\circ}$

Figure 4. Small-angle laser light scattering patterns of the concentrated solutions of PLL $\cdot \mathrm{HBr}$ : Upper, the $H_{\mathrm{V}}$ patterns; lower, the $V_{\mathrm{v}}$ patterns. Polymer concentration in wt\%: a, 48 (the solution was sheared up and down.); b, 60; c, 70, d, 75 . 


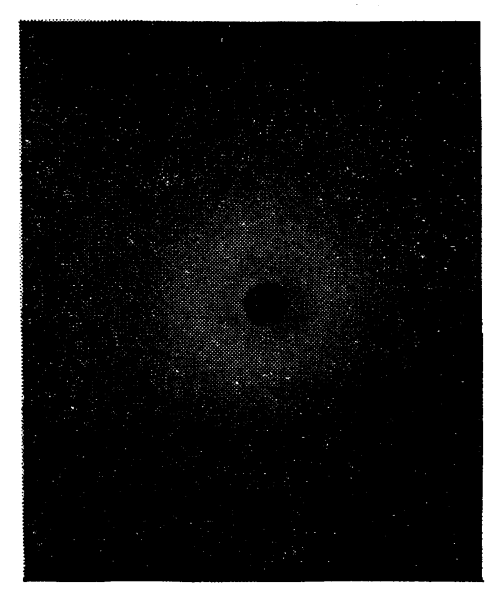

$a$

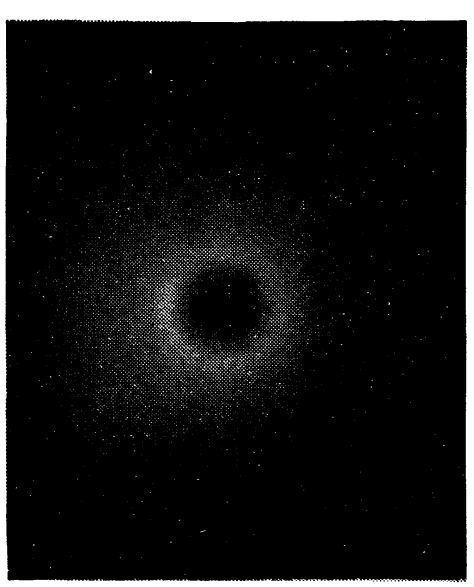

b

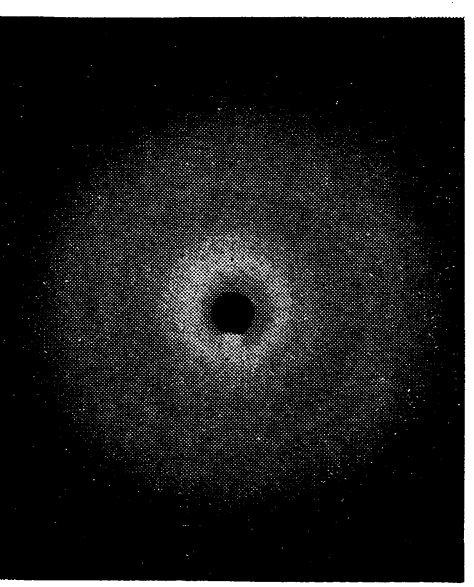

c

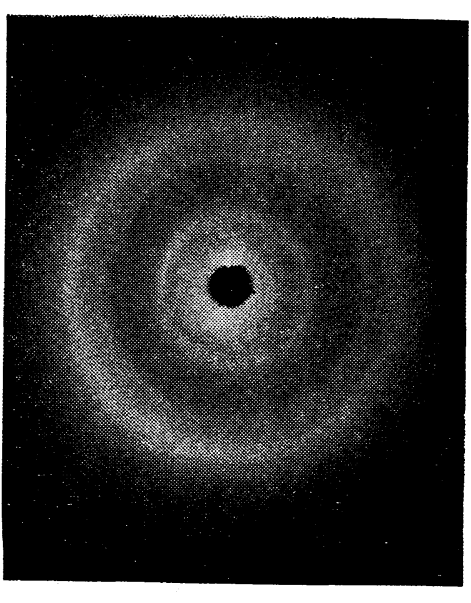

d

Figure 5. X-ray diffraction photographs of the concentrated solutions of PLL·HBr. Polymer concentration in wt \%: a, 40; b, 48; c, $75 ; \mathrm{d}, 85$. 
cular clusters align themselves with their long axes parallel to the shear direction. ${ }^{7,10}$ At the polymer concentration of $75 \mathrm{wt} \%$, the solution begins to scatter light irregularly, corresponding to the possible formation of some fine (semi) crystallites in solution (Figure 4, d).

The X-ray diffraction photograph of the isotropic solution shows an amorphous pattern because there is no ordered structure in solution, whereas the birefringent solutions show Debye-Scherrer rings, suggesting the formation of some ordered structure in solution (Figure 5). The pattern of the birefringent solution changes with increasing polymer concentration. At $48 \mathrm{wt} \%$ the solution has only one, broad ring corresponding to a 13-A spacing which is possibly related to a hexagonal packing of the $\alpha$-helical molecules. At $75 \mathrm{wt} \%$ more rings that are sharp appear, and at a still higher concentration $(85 \mathrm{wt} \%)$ the innermost ring related to the helical conformation disappears and the $3 \mathrm{rd}$ ring from the outermost ring becomes more marked. This Debye-Scherrer ring corresponds to a 4.7-A spacing which is most likely related to the backbone spacing of the polypeptide molecules in the $\beta$ form. ${ }^{11}$ The intensity of this diffraction is not so strong in a $75-\mathrm{wt} \%$ solution, suggesting that this solution is a mixture of the helical and $\beta$ structures. In polymer films of $\mathrm{PLL} \cdot \mathrm{HBr}$, the polypeptide molecules were in the $\alpha$-helical conformation when the films were prepared by drying the isotropic solution very quickly, whereas they were in the $\beta$ form when the films were made very slowly from the birefringent solution. From the microscopic observations, it was known that the isotropic solution passed through the birefringent state when being dried up. These results suggest that the coil-to-helix transition is rather of the instantaneous type and that the helixto- $\beta$ transition is time-dependent.

All this experimental evidence indicates that the birefringent solution of $\mathrm{PLL} \cdot \mathrm{HBr}$ is liquid crystalline at least when its polymer concentration is not so high, say less than $70 \mathrm{wt} \%$ at room temperature. The presence of rod-like molecular clusters that form liquid crystals suggests that these liquid crystals are of the nematic type. The liquid crystals are quite similar to those found in PGA$\mathrm{Na}^{1} \quad$ The formation of liquid crystals immediately follows the transition from the coil to the $\alpha$-helical conformation and there is no intermediate state where the isotropic and birefringent phases coexist. The fact that the critical polymer concentration, at which the transition from the liquid crystalline state to some (semi) crystalline state occurs, is difficult to determine makes it impossible to complete the phase diagram.

\section{EXPERIMENTAL}

The PLL. HBr used in this study was of mean degree of polymerization 890 and was donated by Professor T. Hayakawa of this Institute. It was dissolved in deionized water and introduced into 1-mm quartz cells with a quartz spacer inside in each cell to obtain the desired pathlengths of $0.1 \mathrm{~mm}$ or less. The apparent $\mathrm{pH}$ of the solutions (40-65 wt \%) was about 3.0. Polarizing micrographs and small-angle laser light scattering patterns of the solutions were measured in these optical cells. Infrared absorption spectra oft he solutions were taken between two $\mathrm{CaF}_{2}$ plates and the wide-angle $\mathrm{X}$-ray diffraction photographs in glass capillary tubes of diameter $1 \mathrm{~mm}$. Details of the measurements have been described in a previous paper. ${ }^{1}$ The measurements were carried out at room temperature unless otherwise stated.

Acknowledgments. We wish to thank Professor T. Hayakawa for the donation of the polypeptide specimen used in this study. Thanks are also due Mr. H. Yamagishi for carrying out parts of the measurements.

\section{REFERENCES}

1. E. Iizuka, Y. Kondo, and Y. Ukai, Polym. J., 9, 135 (1977).

2. T. Miyazawa and E. R. Blout, J. Am. Chem. Soc., 83, 712 (1961).

3. P. K. Sarker and P. Doty, Proc. Natl. Acad. Sci., 55, 981 (1966).

4. E. lizuka and J. T. Yang, Biochemistry, 4, 1249 (1965).

5. S. Ya Frenkel', L. G. Shaltyko, and G. K. Elyashevich, J. Polym. Sci., Part C, No. 30, 47 (1970).

6. M. B. Rhodes and R. B. Stein, J. Polym. Sci., Part $A-2,7,1539$ (1969).

7. E. Iizuka, T. Keira, and A. Wada, Mol. Cryst. Liq. Cryst., 23, 13 (1973). 
8. E. Iizuka, Biochim. Biophys. Acta, 243, 1 (1971).

9. E. Iizuka, Adv. Polym. Sci., 20, 79 (1976).

10. E. Iizuka, Proc. 6th Int. Liq. Cryst. Conf., Part D,
Mol. Cryst. Liq. Cryst., 42, 1077 (1977).

11. F. J. Padden, J. H. D. Keith, and G. Giannoni, Biopolymers, 7, 793 (1969). 